\title{
Current instabilities in the pulsar magnetosphere
}

\author{
Axel Jessner ${ }^{1}$, Harald Lesch ${ }^{2}$ and Michael Kramer ${ }^{1}$ \\ ${ }^{1}$ Max Planck Institute for Radio Astronomy, Auf dem Hügel 69, D-53121, Bonn, Germany \\ email: jessner@mpifr-bonn.mpg.de \\ ${ }^{2}$ Universitäts-Sternwarte München, Scheinerstr. 1, D-81679 München, Germany \\ email: lesch@usm.uni-muenchen.de
}

\begin{abstract}
Pulsars are rotating neutron stars with strong magnetic dipole fields $\left(B=10^{4}\right.$ $10^{9} \mathrm{~T}$ ), and high induced surface potentials (ca. $10^{14} \mathrm{~V}$ ). A strong charged particle current is driven out of the polar cap. It returns along an equatorial current sheet. The total dissipated power of the current system is a significant fraction of the observed spin-down power of the pulsar. The Pierce instability occurs when particles are constrained to move in only one dimension and the field from the accumulated space charge exceeds the accelerating background field. Relativistic particle motion enhances the instability which forms narrow regions $(\mathrm{cm})$ of high particle densities and low velocities separated by much longer but more tenuous relativistic flows. The calculated spectrum, power budget and time scales of the magnetospheric Pierce instabilities match the observed radio properties of pulsars.
\end{abstract}

Keywords. (stars:) pulsars: general, acceleration of particles, instabilities.

A strong charged particle current is driven out of the polar caps of a pulsar as a consequence of its rotation and its high surface magnetic field. Contopoulos et al. (1999) has found that the current will return along an equatorial current sheet. Observed pulsar braking indices are not compatible with energy losses arising from waves caused by magnetic dipole rotation. Some pulsars have their magnetic and spin axes very closely aligned. Their dipole spin-down losses would consequently be very small, compared to what is observed. Furthermore, observations of the difference in spin-down losses of PSR $\mathrm{B} 1931+24$, a pulsar with intermitting radio emission find their natural and very accurate explanation in an intermitting current with the co-rotational charge density dissipating the polar cap potential difference (Kramer et al., 2006). For PSR B1931+24 the current is responsible for a third of the spin down losses.

Therefore a net non-neutral current is expected to flow across a net potential difference in order to provide for the electrical spin-down loss that occurs in addition to the dipole radiation loss. Komissarov (2006) has suggested, that losses in the current sheet may convert a significant amount of spin-down energy into optical and $\gamma$-ray emission, but the origin of the required resistivity, or the stability of the magnetospheric current system and its implication for pulsar radio emission have not been scrutinized. One of the basic assumptions of MHD simulations is the vanishing of $E_{\|}$along the flow and that makes it difficult to derive other than ohmic losses.

Solving the equation of motion for particles along the magnetic field lines self-consistently under consideration of their losses by inverse Compton scattering and curvature radiation can provide estimates of particle energies and losses when the force-free constraints are violated (Jessner et al. 2001). This approach provides a natural explanation for the acceleration of particles to high energies, giving rise to X-ray and optical emissions and perhaps pair creation. 


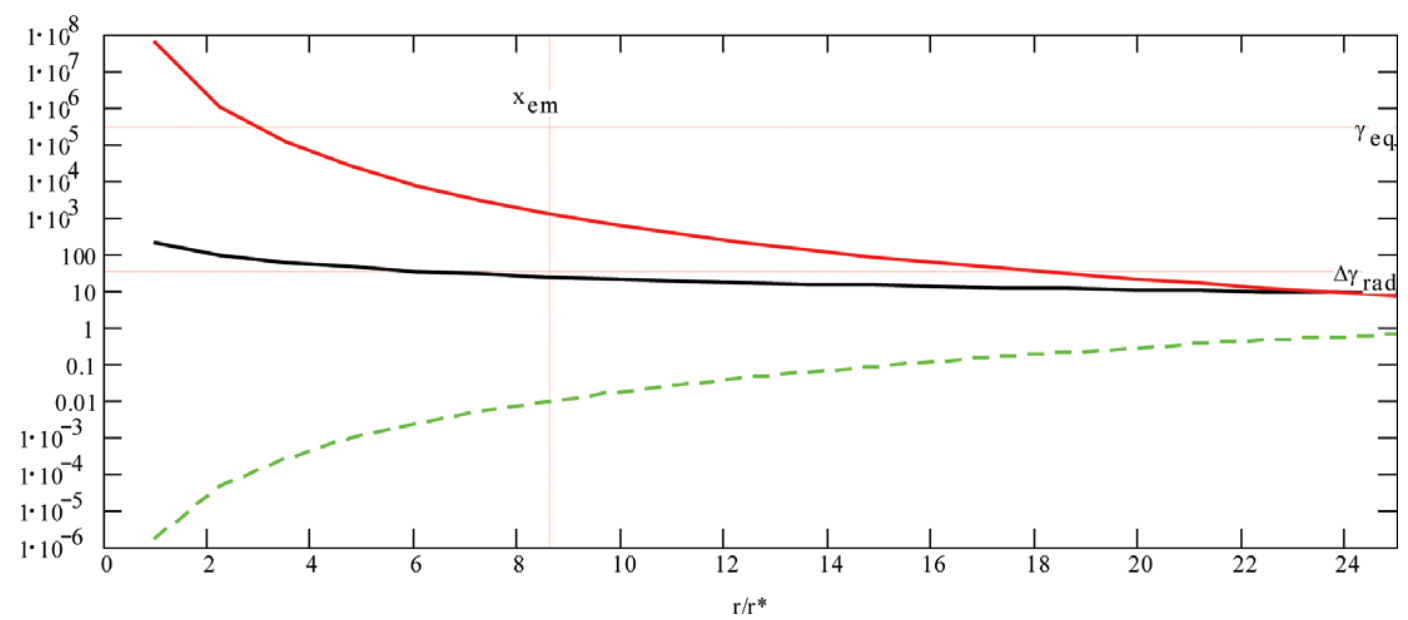

Figure 1. Characteristics of the Pierce instability on the critical field line of PSR B $2021+51$ as a function of distance from the neutron star. Black: Pierce length $L_{p}[\mathrm{~m}]$, red: max. Lorentz factor $\gamma_{\max }$, green dashed: thickness of virtual cathode layer $L_{v c}[\mathrm{~m}]$. The estimated location of the emission region $x_{e m}$ and the Lorentz factor corresponding to the radio emission losses $\Delta \gamma_{\text {rad }}$ are also indicated.

However, by omitting to solve Poisson's equation for the self-field and densities of the streaming particles at the same time, such modelling will fall short of being a true representation of the actual conditions in the pulsar magnetosphere.

Non-neutral currents can become unstable, especially when constrained to move in only one dimension by a strong magnetic field. This was noticed by Pierce (1949) in experiments with strong electron beams in a magnetic field. Davidson (2001) gives a comprehensive treatment of the space charge limited flow problem and provides solutions for different geometrical configurations and particle energies. The existence of a Pierce-type instability in pulsar magnetospheres had first been described by Mestel et al. (1985). Jessner et al. (2002) and Beloborodov (2008) discussed the existence of the Pierce instability in pulsar magnetospheres.

Particles start from the neutron star with thermal velocities where a cathode is formed in a boundary layer close to the surface when the charge density $n_{0}$ is sufficient to compensate the surface electric field. The particles will initially be non-relativistic $(v=$ $\left.\beta_{0} c\right)$ but the strong electric field will quickly accelerate them to relativistic energies and the charge density of the current will decrease to about $\beta_{0} q_{e} n_{0}$ by virtue of current conservation. Hence a thermal emission current with a non-relativistic co-rotation surface density cannot compensate the strong parallel electric field as soon as charges become relativistic after leaving the cathode layer. The particles will continue to gain energy until their space charge is sufficient to compensate the background $E_{\|}$. From then on, their own field will repel them and they loose energy until they come to a stop and a new 'virtual' cathode is formed. The cycle will repeat, but as for a dipolar field $E_{\|} \propto r^{-4}$ and the current density $j \propto r^{-3}$ these oscillations will end at a certain distance from the neutron star and the flow will cease to be relativistic.

A virtual cathode will form whenever the space charge electric field grows faster than the external electric field. A homogeneous charged relativistic beam, having constant charge density and a linearly growing space-charge field forms a virtual cathode when the background electric field is only slowly changing. In that case the virtual cathode will form at a distance of $L_{p}=4 \frac{\epsilon_{0} E_{\|}}{q_{e} n_{e}}$ and the maximum Lorentz factor will be given 
by $\gamma_{\max }=2 \frac{\epsilon_{0} E_{\|}^{2}}{n_{e} m_{e} c^{2}}$ as the equilibrium between the energy density of the background field and the current energy density $\gamma_{\text {max }} n_{e} m_{e} c^{2}$. Although $L_{p}$ grows with $E_{\|}$, the width of the non-relativistic virtual cathode region $L_{v c}=\frac{m_{e} c^{2}}{q_{e} E_{\|}}$decreases with $E_{\|}$and in the non-relativistic regime $L_{p}$ and $L_{v c}$ become comparable.

The pulsar B2021 +51 with period $P=0.529 \mathrm{~s}$ and surface magnetic field of $B_{0}=1.3 \times$ $10^{8} \mathrm{~T}$ serves as an illustrative example. For the pulse width of $10^{\circ}$ at $1.4 \mathrm{GHz}$ we obtain an emission height of $x_{e m}=8.7 \times r_{n s}$ and a background electric field $E_{\|}$of $5.4 \times 10^{9} \mathrm{~V} / \mathrm{m}$ together with a current density of $2.5 \times 10^{4} \mathrm{~A} / \mathrm{m}^{2}$. We compute numerical solutions for the set of coupled differential equations (Poisson's equation and the relativistic equation of motion)

$$
\frac{d E}{d z}=-\frac{\kappa I_{t o t}}{A_{e m} \epsilon_{0} c|\beta|} \text { and } \frac{d}{d z} \beta \gamma=\frac{q_{e}}{m_{e} c^{2}}\left(E-(1-\kappa) E_{\|}\right)
$$

assuming a charge density of $99 \%(\kappa=0.99)$ of the full equilibrium value, which will shield the background $E_{\|}$by a factor of $1-\kappa$. Background $E_{\|}$and current density $j=\frac{I_{t o t} \cdot \kappa}{c A_{e m}}$ vary slowly on the scales of the instability, justifying a $1-\mathrm{D}$ cartesian coordinate approximation. The numerical results agree with the derived values for $\gamma_{\max }, L_{p}$ and $L_{v c}$. Fig. 1 shows the variation of $\gamma_{\max }, L_{p}$ and $L_{v c}$ along the outermost open field line which still has an accelerating background field all the way out to the light cylinder. For the emission region we find $L_{v c}=1 \mathrm{~cm}$, a repetition of the pattern after $L_{p}=23 \mathrm{~m}$ with a maximum Lorentz factor of $\gamma_{\max }=1234$ which is about fourty times the amount of $\Delta \gamma_{\text {rad }}=33.6=\frac{L_{\text {rad }} q_{e}}{I_{\text {tot }} m_{e} c^{2}}$ that needs to be dissipated by the beam particles to yield the observed radio luminosity of $L_{\text {rad }}=5.8 \times 10^{18} \mathrm{~W}$. The space charge electric field peaks in the narrow virtual cathode regions where the density is also at its maximum, giving rise to strong pulses of sub-nanosecond $\tau>L_{v c} / c=10^{-11} \mathrm{~s}$ duration. Such Nano-shots are observed in giant pulses from the Crab pulsar. and there are indications of regular patterns of narrow emission regions separated on scales of a few $\mathrm{m}$ in Crab high-frequency giant pulses (Jessner et al. 2010). Loehmer et al. (2008) have shown that the superposition of sub-nanosecond emissions can also provide an explanation for the observed form of pulsar spectra. Our described solutions are stationary, but highly sensitive to the value of $\frac{1-\kappa}{\kappa}$ and we expect small variations of the current density to cause large fluctuations in the local radio emission.

\section{References}

Beloborodov, A. M. 2008, ApJ, 683, L41-44

Davidson, 2001, Physics of non-neutral beams, Imperial College Press London 2001

Komissarov, S. S. 2006, MNRAS, 367, 19

Kramer, M., Lyne, A. G., O'Brien, J. T., Jordan, C. A., \& Lorimer, D. R. 2006, Science, 312, 549

Contopoulos, I., Kazanas, \& D. Fendt, C. 1999, ApJ, 511, 351

Jessner, A., Lesch, H. \& Kunzl, T. 2001, ApJ, 547, 959

Jessner, A., Lesch, H. \& Kunzl, T. 2002, 270. WE-Heraeus Seminar on Neutron Stars, Pulsars and Supernova Remnants. (Eds.) W. Becker et al. MPE-Report No. 278, 209-214

Jessner, A., Popov, M. V., Kondratiev, V. I., Kovalev, Y. Y., Graham, D., Zensus, A., Soglasnov, V. A., Bilous, A. V., \& Moshkina, O. A. 2010, A\& in press

Loehmer, O., Jessner, A., Kramer, M., Wielebinski, R. \& Maron, O. 2008, A\&GA, 480, 623

Mestel, L., Robertson, J. A., Wang, Y. M. \& Westfold, K. C. 1985, MNRAS, 217, 443

Pierce, J. R. 1949, The Theory and Design of Electron Beams, van Nostrand, (Toronto, New York, London 1949) 